\title{
Probiotic supplementation in neonates with congenital gastrointestinal surgical conditions: a pilot randomised controlled trial
}

\author{
Shripada Rao ${ }^{1,2,3 凶}$, Meera Esvaran ${ }^{4}$, Liwei Chen ${ }^{5}$, Anthony D. Keil ${ }^{6}$, Ian Gollow ${ }^{7}$, Karen Simmer ${ }^{1,2,3}$, Bernd Wemheuer ${ }^{4,8}$, \\ Patricia Conway ${ }^{4,5}$ and Sanjay Patole $e^{1,2,3}$ \\ (c) Crown 2021
}

\begin{abstract}
OBJECTIVE: To evaluate whether probiotic supplementation attenuates gut-dysbiosis in neonates with congenital gastrointestinal surgical conditions (CGISC).

METHODS: Sixty-one neonates ( $\geq 35$ weeks gestation) with CGISC were randomised to receive daily supplementation with a triple-strain bifidobacterial probiotic $(n=30)$ or placebo $(n=31)$ until discharge. Stool microbiota was analysed using $16 \mathrm{~S}$ ribosomal RNA gene sequencing on samples collected before (T1), 1 week (T2), and 2 weeks (T3) after supplementation and before discharge (T4). The primary outcome was the sum of the relative abundance of potentially pathogenic families of Clostridiaceae, Enterobacteriaceae, Enterococcaceae, Pseudomonaceae, Staphylococcaeae, Streptococcaceae, and Yersiniaceae at T3.

RESULTS: The median gestational age [38 weeks (IQR: 37.1-38.9)] was similar in both groups. The probiotic group had lower rates of caesarean deliveries ( $40 \%$ versus $70 \%, p=0.02)$. The relative abundance of potentially pathogenic families was lower in the probiotic group compared to placebo at T3 [(median: 50.4 (IQR: 26.6-67.6) versus 67.1 (IQR: 50.9-96.2); $p=0.04$ ). Relative abundance of Bifidobacteriaceae was higher in the probiotic group at T3 [(median: 39.8 (IQR: 24.9-52.1) versus 0.03 (IQR $0.02-2.1) ; p<0.001)$. Stratified analysis continued to show a higher abundance of Bifidobacteriaceae in the probiotic group, irrespective of the mode of delivery.

CONCLUSIONS: Probiotic supplementation attenuated gut dysbiosis in neonates with CGISC.

TRIAL REGISTRATION: http://www.anzctr.org.au (ACTRN12617001401347).
\end{abstract}

Pediatric Research (2022) 92:1122-1131; https://doi.org/10.1038/s41390-021-01884-x

IMPACT:

- Probiotic supplementation attenuates gut dysbiosis and improves stool short-chain fatty acid levels in neonates with congenital gastrointestinal surgical conditions.

- This is the second pilot RCT of probiotic supplementation in neonates with congenital gastrointestinal conditions.

- These findings will pave the way for conducting multicentre RCTs in this area.

\section{INTRODUCTION}

The common morbidities in neonates with congenital gastrointestinal surgical conditions (CGISC) are feed intolerance and healthcareassociated infections (HAl). ${ }^{1-9}$ Recurrent administration of antibiotics, delayed enteral feeds, use of parenteral nutrition (PN), and delayed exposure to the mother's skin and breast milk microbiota can lead to intestinal dysbiosis in these infants. ${ }^{10-15}$ Our previous prospective study found that neonates with CGISC develop gut dysbiosis during their stay in the neonatal intensive care unit. ${ }^{13}$
Experimental studies have shown that probiotic supplementation attenuates gut dysbiosis, strengthens the gut barrier, prevents enteropathogenic infections, reduces antimicrobial resistance, enhances immunity, and promotes gut peristalsis. ${ }^{16}$ Through these mechanisms, probiotics have the potential to improve the outcomes of neonates with CGISC. ${ }^{16}$ Many beneficial biological functions of probiotics are mediated via short-chain fatty acids (SCFAs). ${ }^{17}$ The major SCFAs $(80-95 \%)$ in the gut are acetate, propionate, and butyrate. ${ }^{18,19}$

\footnotetext{
${ }^{1}$ Neonatal Intensive Care Unit, Perth Children's Hospital, Perth, WA, Australia. ${ }^{2}$ Neonatal Intensive Care Unit, King Edward Memorial Hospital for Women, Perth, WA, Australia.

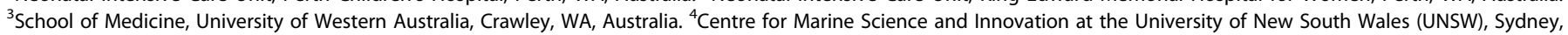

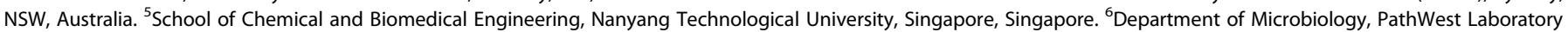

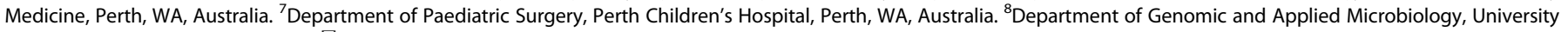
of Göttingen, Göttingen, Germany. ${ }^{凶}$ email: shripada.rao@health.wa.gov.au
} 
In infants, SCFAs are produced by the fermentation of milk oligosaccharides by gut bacteria, and the type of milk has a significant effect on the composition of the SCFA. ${ }^{20}$

Our previous prospective study also found that neonates with CGISC have lower faecal SCFA levels. ${ }^{13}$

Systematic reviews from adult studies have concluded that probiotic/synbiotic supplementation is safe and reduces the risk of postoperative infections. ${ }^{21}$ A pilot randomised controlled trial (RCT) $(n=24)$ in neonates with gastroschisis reported that gut dysbiosis was partially attenuated by the probiotic Bifidobacterium longum subsp. infantis. ${ }^{22}$ Our systematic review found limited evidence regarding the role of probiotics in neonates with CGISC and recommended the conduct of RCTs. ${ }^{23}$ Hence, we conducted this pilot RCT to evaluate the efficacy and safety of probiotics in attenuating dysbiosis and improving SCFA levels in term and nearterm infants with CGISC.

\section{METHODS}

Hypothesis

Probiotic supplementation attenuates gut dysbiosis and increases stool SCFA levels in term and near-term neonates with CGISC.

\section{Design and setting}

Double-blind RCT in the neonatal intensive care unit of Perth Children's Hospital, Western Australia. Approval was obtained from the Institutional Human Research Ethics Committee (HREC Ref Number RGS0000002554). It was registered with the Australia and New Zealand Clinical Trials Registry (ACTRN12617001401347). The protocol is available in Supplement File 3. Infants were recruited between November 2017 and March 2020.

\section{Eligibility criteria included}

Neonates ( $\geq 35$ weeks' gestation) with intestinal atresia, malrotation, congenital diaphragmatic hernia (CDH), tracheoesophageal fistula (TOF), gastroschisis, exomphalos, Hirschsprung disease, imperforate anus, short bowel syndrome, and other surgical conditions requiring stomas (e.g., severe meconium ileus, microcolon). Preterm infants less than 35 weeks of gestation were excluded to reduce the confounding effect of prematurity on the gut microbiota.

\section{Intervention}

A mixture of three strains (Bifidobacterium breve M-16V, Bifidobacterium longum subsp. infantis $\mathrm{M}-63$, and Bifidobacterium longum subsp. longum BB536; $1 \times 10^{9}$ colony-forming units (CFU) of each strain per $1 \mathrm{~g}$ sachet; Morinaga Milk Industry Co., Ltd., Japan]. Dose was 1 sachet per day, which provided a total of $3 \times 10^{9}$ (i.e., 3 billion) CFU per day. Placebo: Maltodextrin. The trial supplements were stored in a refrigerator at $2-8^{\circ} \mathrm{C}$. Group assignments were allocated using a computer-generated sequence in randomly ordered block sizes of 2 and 4 . These sequences were generated by our institutional trial pharmacist, without the involvement of the research team. Allocation concealment was optimised through pharmacy-controlled allocation; the clinical trial pharmacist generated random numbers and used them to prepare sequentially numbered individual boxes, each containing 30 sachets of trial supplements. The sachets containing probiotics or placebo were of identical design, weight, and volume; the boxes containing the sachets were also of similar design. The contents of the sachets (probiotic or placebo) were similar in texture, smell, and taste. All these steps were undertaken to ensure adequate blinding of healthcare providers and parents. Once parental consent was obtained, the chief investigator or his delegate allocated the next box of study supplement to the infant without knowledge of the contents of the box (probiotic or placebo).

In the postoperative period, once baseline stool samples were collected, infants were given trial supplements once daily until discharge. Trial supplements were dissolved in $1.5 \mathrm{~mL}$ of expressed breast milk (EBM) or sterile water (if EBM was not available) and administered via the feeding tube or mouth. Supplementation was continued even when the infants did not receive enteral feeds. If an infant was having continuous or intermittent gastric suctioning, once the trial supplements were given, suctioning was stopped for 3-4 $\mathrm{h}$ prior to recommencing.

\section{Feeding regimen of study infants}

The standard policy of our unit is to commence PN within $48-72 \mathrm{~h}$ of admission for surgical infants who are unable to tolerate enteral feeds. Enteral feeding with expressed breast milk were commenced as soon as possible in the postoperative period (usually on postoperative day 2 and advanced as tolerated), depending on the underlying surgical condition and consensus opinion of neonatologists and surgeons. Infant formulae or hydrolysed formulae are rarely used in our unit for surgical infants. None of our study infants received donor breast milk because it was reserved for preterm infants less than 32 weeks of gestation in our unit.

\section{Stool sample collection, storage, and analysis}

Stool samples were collected at four time points: (a) as soon as possible after admission, but before commencing trial supplements (T1); (b) 1 week (T2), and (c) 2 weeks after commencing supplements (T3); and (d) prior to discharge (T4).

The samples were collected from the nappies into sterile microvials and stored in the NICU at $-20^{\circ} \mathrm{C}$ for $3-4$ days and subsequently at $-80^{\circ} \mathrm{C}$. At the completion of full recruitment, samples were shipped on dry ice to the University of New South Wales (Sydney, Australia), where microbial analysis was undertaken. Acidified samples were frozen at $-20^{\circ} \mathrm{C}$ and shipped to the School of Chemical and Biomedical Engineering, Nanyang Technological University, Singapore, where SCFA analysis was performed.

The stool microbiota was assessed using the $16 \mathrm{~S}$ ribosomal RNA gene sequencing method (Supplementary File 1). Stool short-chain fatty acid assay was performed according to our previously described method ${ }^{13}$ with slight modifications (Supplementary File 1). The scientists who conducted microbial and SCFA assays and their respective statistical analyses were blinded to the randomisation groups. They conducted statistical analyses of data from stool samples as group 1 versus group 2. The trial pharmacist disclosed the groupings only after receiving all the results through e-mail. This ensured adequate blinding of clinicians, research teams, lab scientists, and statisticians throughout all stages of the trial.

\section{Primary outcome}

The primary outcome was the sum of the relative abundance of potentially pathogenic families of Clostridiaceae, Enterobacteriaceae, Enterococcaceae, Pseudomonaceae, Staphylococcaeae, Streptococcaceae, and Yersiniaceae at T3 using 16S ribosomal RNA gene sequencing methods. T3 was chosen as the main time point of interest because 2 weeks is a reasonable duration of supplementation to enable colonisation by the probiotic strains, and many study infants will still be in the hospital.

\section{Secondary outcomes}

(1) Stool microbiota at T1, T2, and T4 time points; (2) SCFA levels at all time points: (3) short-term clinical outcomes during initial stay in the NICU, such as incidence of mortality, HAl, duration of antibiotics, PN, hospital stay, time to reach full feeds after surgery, and physical growth. The $z$-scores for weight, length, and head circumference at birth were calculated using the Fenton growth charts ${ }^{24}$ and at discharge using the WHO charts ${ }^{25}$ through the publicly accessible PediTools website of clinical calculators. ${ }^{26}$

\section{Statistical considerations}

Sample size estimation. In a recent $\mathrm{RCT}$, the sum of the relative abundance of potentially pathogenic families such as Enterobacteriaceae, Staphylococcaeae, Enterococcaceae, Clostridiaceae, and Streptococcaceae was approximately $76 \%$ in the stools of infants with gastroschisis who received placebo.22 Hence, we calculated that a total sample size of 60 infants (probiotic: 30 , placebo: 30 ) would be required to demonstrate a $50 \%$ reduction of potentially pathogenic bacterial families from 76 to $38 \%$ after 2 weeks of supplementation with probiotics with an alpha error of 0.05 , and a power of $80 \%$.

Statistical analysis of clinical data. Continuous data with normal distribution were summarised using mean and standard deviations (SD) and compared using the two-sample $t$-test. Median, interquartile range (IQR), and range were used to summarise data with skewed distribution and compared using the Wilcoxon rank-sum test. Binary outcomes were compared using the Fisher's exact test. To compare the $z$-scores of physical growth parameters at discharge versus birth, the matched pairs $t$-test was used. For all analyses, a $p$ value less than 0.05 was considered significant. 


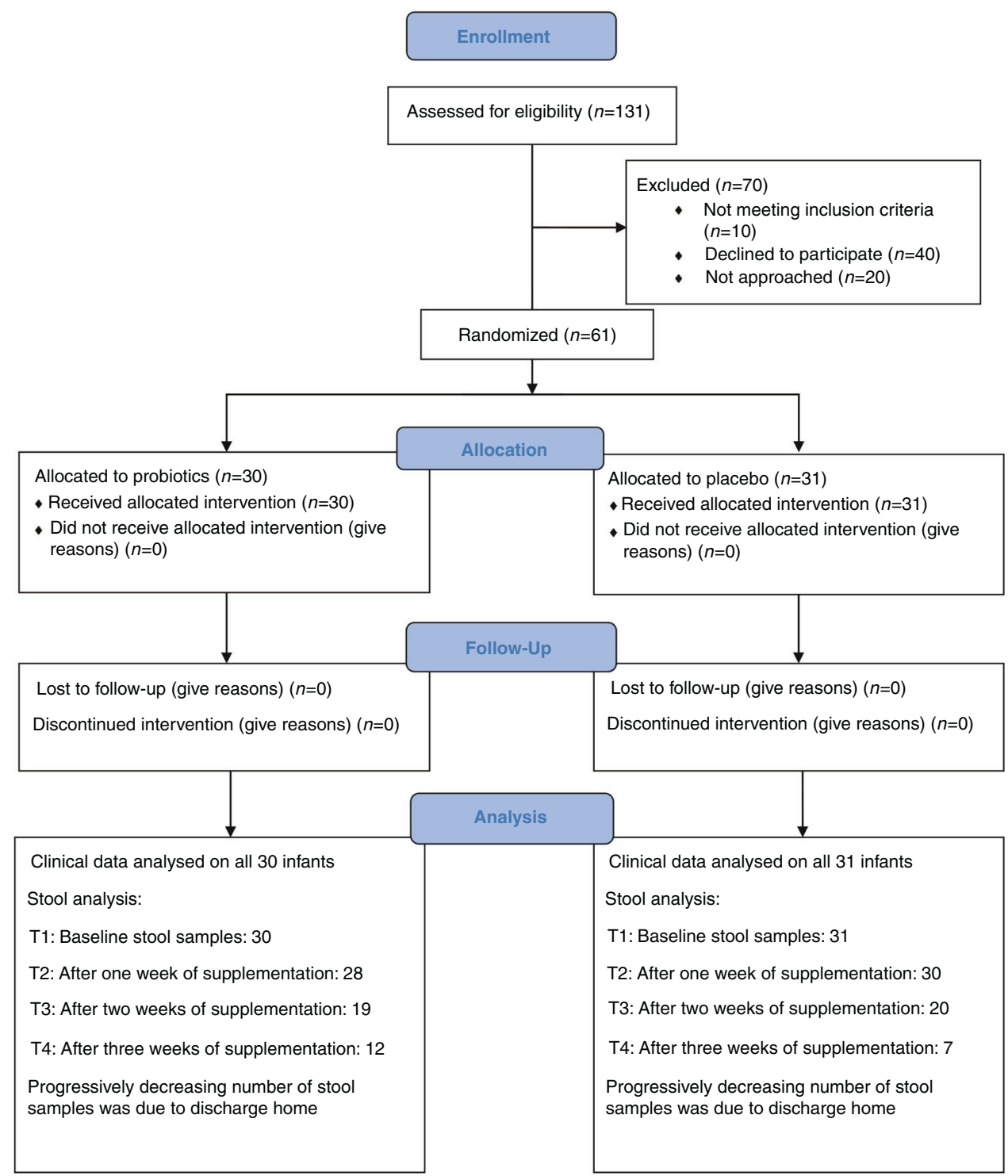

Fig. 1 CONSORT flow diagram showing participant flow through the trial.

Statistical analysis for microbiological data. Analyses were conducted with $\mathrm{R}$ version 3.6.0. For microbial richness, linear mixed model effects (LME) test (MASS, Ime4, and ImerTest packages) was used to identify if there were significant differences between the groups over time. In our LME model, Subject ID was a random factor, while time and treatment were used as fixed factors. Post hoc pairwise comparisons were performed using Tukey's HSD method to adjust for multiple comparisons. Wilcoxon rank-sum test with Benjamini-Hochberg correction to adjust for multiple testing was used to identify differences between the groups at the various time points.

For beta diversity, PERMANOVA was used to check if community structures differed between the groups at the various time points followed by pairwise.adonis test (https://github.com/bwemheu/pairwise. adonis) for pairwise comparisons between the groups. $P$ values were adjusted for multiple testing using the Benjamini-Hochberg correction.

Taxa significantly different between the groups were identified at the phylum, class family, and genus levels using analysis of the analysis of the composition of the microbiome (ANCOM; v2.0). ${ }^{27}$ ANCOM compares the logratio abundance of each taxon among multiple groups to all the remaining taxa. It uses the Kruskal-Wallis test to assess significant differences, with multiple testing corrected by the Benjamini-Hochberg (BH) false discovery rate. For this study, we accepted the $W$-statistic cutoff of 0.7 from the ANCOM output to define taxa as significantly different. Pairwise comparisons in the groups over time was then determined using our LME model. For pairwise comparison of the taxa at $\mathrm{T} 3$, Wilcoxon rank-sum test with $\mathrm{BH}$ correction was used. For all microbiota and SCFA analysis an adjusted $p$ value of $<0.05$ was considered significant.

Statistical analysis of SCFA data. Wilcoxon's rank analysis with BH correction was performed to compare SCFA concentrations between the two groups (probiotics and placebo) at the different time points.

An independent data safety and monitoring committee (DSMC) reviewed the data at $50 \%$ of recruitments and advised to continue the study. The safety issues that arose during the early stages of the trial are described in Supplementary File 1. The CONSORT checklist (Supplementary File 2) was used to report the results of this $\mathrm{RCT}^{28}$

\section{RESULTS}

The study recruitment was completed in March 2020 after achieving full sample size. A total of 61 infants were randomised to receive either probiotics $(n=30)$ or placebo $(n=31)$. All infants received the trial supplements as per original randomisation without any crossovers. All infants were analysed as per the original assigned groups. Figure 1 shows a CONSORT flow diagram. Table 1 presents the clinical details of the study infants. The median gestational age, birth weight, Apgar scores, cord $\mathrm{pH}$, and lactate levels were similar between the two groups. The incidence of maternal pregnancy- 
Table 1. Clinical characteristics of study infants.

\begin{tabular}{|c|c|c|c|}
\hline & Probiotic $(N=30)$ & Placebo $(\mathbf{N}=\mathbf{3 1})$ & $P$ value \\
\hline Gestation (weeks) & 38.1 (IQR: 37.1-39) (range: 35.1-41) & 37.7 (IQR: 37.1-38.9) (range: 35.6-41.8) & 0.675 \\
\hline Birth weight (g) & 2960 (IQR: 2570-3688) (range: 1885-4130) & 2985 (IQR: 2570-3270) (range: 2000-3730) & 0.531 \\
\hline Birth length $(\mathrm{cm})$ & 50 (IQR: 47-51) (range: 40-57) & 49 (IQR: 47-50) (range: 43-56) & 0.623 \\
\hline Birth head circumference $(\mathrm{cm})$ & 33.7 (IQR: 32.5-35) (range: 31-37) & 34 (IQR: 33-35) (range: 31-37.5) & 0.820 \\
\hline Maternal PIH & 0 & $1(3 \%)$ & 1.000 \\
\hline Maternal APH & $7(23.3 \%)$ & 0 & 0.005 \\
\hline Maternal chorioamnionitis & 0 & 0 & NE \\
\hline Maternal intrapartum antibiotics & $18(60 \%)$ & $20(64 \%)$ & 0.934 \\
\hline Caesarean & $12(40 \%)$ & $22(71 \%)$ & 0.021 \\
\hline Apgar scores at $5 \mathrm{~min}$ & 9 (IQR: 9-9) (range: 7-10) & 9 (IQR: 8-9) (range: 6-10) & 0.768 \\
\hline Cord $\mathrm{pH}$ & 7.27 (IQR: 7.23-7.31) (range: 7.15-7.40) & 7.28 (IQR: 7.23-7.33) (range: 7.1-7.36) & 0.862 \\
\hline $\mathrm{CDH}$ & 7 & 1 & 0.026 \\
\hline Day of life, first stool sample & 6 (IQR: 5-6) (range: 2-17), $N=30$ & 6 (IQR: 3-9) (range: $1-25), N=31$ & 0.425 \\
\hline Day of life, second stool sample & 15.5 (IQR: $13-17.5$ ) (range: $11-27), N=28$ & 13 (IQR: $12-18$ ) (range: $9-34), N=30$ & 0.121 \\
\hline Day of life, third stool sample & 23 (IQR: 20-25) (range: 16-30), $N=19$ & 20 (IQR: 20-26) (range: 13-42), $N=19$ & 0.463 \\
\hline Day of life, fourth stool sample & 38.5 (IQR: 29.5-42) (range: $26-46), N=8$ & 34 (IQR: 32-38) (range: 27-53), $N=6$ & 0.824 \\
\hline Day of life consent was given & 5 (IQR: 4-7) (range: 2-18) & 5 (IQR: 3-7) (range: 2-25) & 0.535 \\
\hline $\begin{array}{l}\text { Day of life supplements were } \\
\text { commenced }\end{array}$ & 6.5 (IQR: 5-9) (range: 4-18) & 7 (IQR: 5-11) (range: 3-26) & 0.820 \\
\hline $\begin{array}{l}\text { Duration of } \\
\text { supplementation (days) }\end{array}$ & 18.5 (IQR: 11-27) (range: 3-54) & 16 (IQR: 10-22) (range: 4-58) & 0.337 \\
\hline
\end{tabular}

supplementation (days)

SVD spontaneous vaginal delivery, AVD assisted vaginal delivery, CS caesarean section, APH antepartum haemorrhage, $P I H$ pregnancy-induced hypertension, $N E$ not estimable, IQR interquartile range, NE not estimable, $C D H$ congenital diaphragmatic hernia, $C D O$ congenital duodenal obstruction, TOF tracheooesophageal fistula, $O A$ oesophageal atresia, JIA jejuno-ileal atresia, CF cystic fibrosis, HD Hirschsprung disease, IQR interquartile range.

induced hypertension (PIH), chorioamnionitis, and peripartum antibiotics was similar between the groups. The incidence of antepartum haemorrhage (APH) was higher, and caesarean delivery rates were lower in the probiotic group. The probiotic group had more infants with congenital diaphragmatic hernia than the placebo group. The median age at surgery was 2 days in both the groups. The median age at commencement of supplementation was approximately 7 days in both groups, and the duration of supplementation was 16-18 days.

\section{Results of microbial analysis}

Of the 61 recruited infants, stool samples were available for 61,58 , 39 , and 29 infants at time points T1, T2, T3, and T4, respectively. The attrition in sample size was because 22 infants were discharged home before completing 2 weeks of supplementation (i.e., before reaching T3). We could not extend the trial beyond March 2020 to recruit additional infants because of severe restrictions that were implemented due to the COVID-19 pandemic during that period.

Alpha diversity (i.e., Richness and Shannon diversity index) was comparable between the two groups at all time points (all $p>$ 0.05; Fig. 2a). Similar results were obtained on Chao1 and ACE estimations (data not shown). Observed OTUs increased in both groups over time and the probiotic group displayed significantly increased bacterial richness at T4 compared to T1 $(p=0.015)$ and
T2 $(p<0.01$; Fig. 2a). Beta diversity analysis revealed that both groups had similar community structures at T1 ( $p=0.807$; Fig. 2b). However, at T2, T3, and T4, the community structures of the probiotic group were significantly different from those of the placebo group (all $p<0.05$; Fig. 2b).

Comparisons at the bacterial phylum level. Actinobacteria, Bacteroidetes, Cyanobacteria, Firmicutes, and Proteobacteria were the most common phyla in the stool samples (e-Fig.1A in Supplementary File 1). The median relative abundance of Actinobacteria was significantly higher in the probiotic group than in the placebo group at T3 $(40.1 \%$ versus $0.1 \% ; p<0.0001)$ and at T2 and T4 (both $p<$ 0.01 ; e-Fig. 1B in Supplementary File 1). Although the relative abundance of Proteobacteria was similar between the two groups at T3 $(22.7 \%$ in probiotic versus $50.3 \%$ in placebo; $p=0.27)$ and at other time points (e-Fig. 2 in Supplementary File 1), the levels in the probiotic group decreased over time, with T2 and T4 being significantly different from T1 ( $p=0.048$ and $p=0.046$, respectively).

Comparisons at the bacterial class level. The relative abundance of Gammaproteobacteria between the two groups was similar at all time points (e-Fig. 3 in Supplementary File 1).

Comparisons at the bacterial family level. The relative abundance of the sum of potentially pathogenic families of Clostridiaceae, 
a
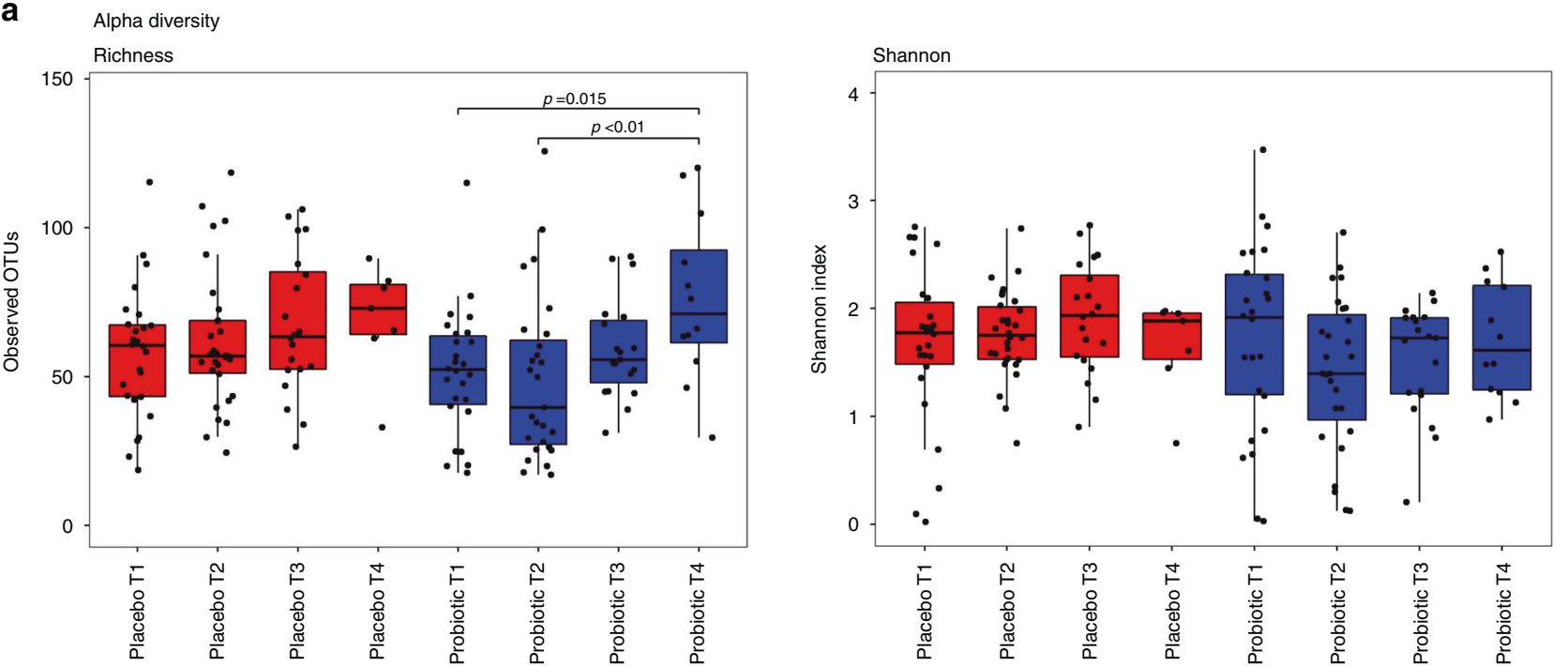

b
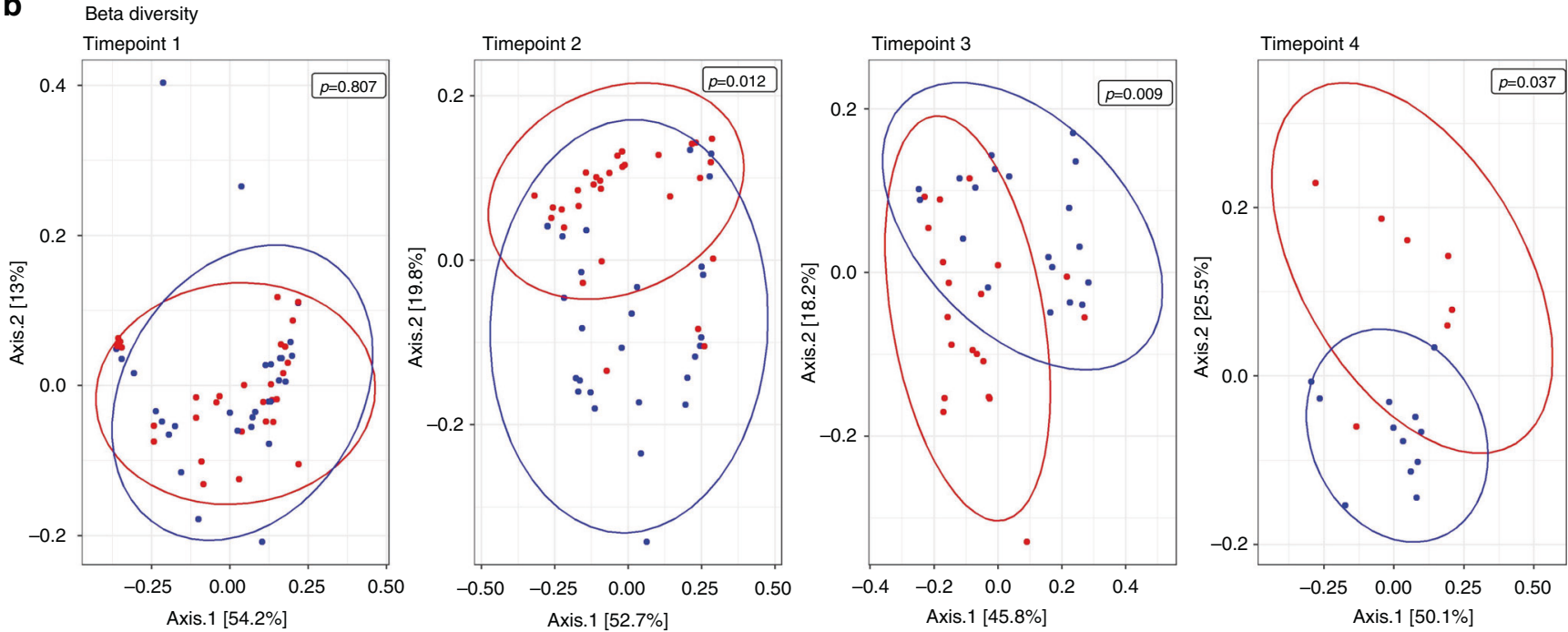

Fig. 2 Alpha and Beta diversity at various time points in the stool samples of study infants. a Alpha diversity. Richness and the Shannon diversity index were similar between the probiotic and placebo groups at all time points (all $p>0.05$ ). b Beta diversity as measured by weighted unifrac by time point. At baseline, infants in the probiotic group had similar community structures to the placebo group ( $p=0.807)$. However, at subsequent time points T2, T3, and T4, the community structures of the probiotic group were significantly different from the placebo group (all $p<0.05$ ).

Enterobacteriaceae, Enterococcaceae, Pseudomonadaceae, Staphylococcaceae, Streptococcaceae, and Yersineaceae were significantly lower in the probiotic group compared to placebo at T3 [(median: 50.4 (IQR: 26.6-67.6) versus 67.1 (IQR 50.9-96.2); $p=$ $0.044)$ and at T2 and T4 ( $p=0.006$ and $p=0.014$, respectively; Fig. 3a). Since there was a slight imbalance in the number of stool samples between the probiotic $(n=19)$ and placebo $(n=20)$ groups at T3, one sample from the placebo group was randomly removed and the data were reanalysed. The results continued to remain similar to the original analysis $(p=0.036)$.

The relative abundance of the family Bifidobacteriaceae was significantly higher in the probiotic group at T3 [(median: 39.8 (IQR: 24.9-52.1) versus 0.03 (IQR 0.02-2.1); $p<0.001$ ) and at T2 and T4 (both $p<0.001$; Fig. 3b).

Comparisons at the bacterial genus level. The relative abundance of the genus Bifidobacterium was significantly higher in the probiotic group at T2, T3, and T4 (all $p<0.001$; e-Fig. 4 in Supplementary File 1).

Stool SCFAs: The total SCFA levels were higher in the probiotic group than in the placebo group at T3 ( $p=0.008$; Fig. 4). Acetate levels were higher in the probiotic group (e-Fig. 5 in Supplementary File 1). The butyrate levels were similar between the groups at all time points except T2, when they were lower in the probiotic group (e-Fig. 6 in Supplementary File 1). The propionate levels were similar between the two groups at all time points (e-Fig. 7 in Supplementary File 1).

Post hoc subgroup analysis based on the mode of delivery Since the probiotic group had significantly lower rates of caesarean section, we conducted a post hoc analysis separately for infants born via caesarean section and through the vaginal route. The results showed a significantly higher abundance of Bifidobacteriaceae in the 

Relative abundance of potentially pathogenic bacterial families (primary outcome)

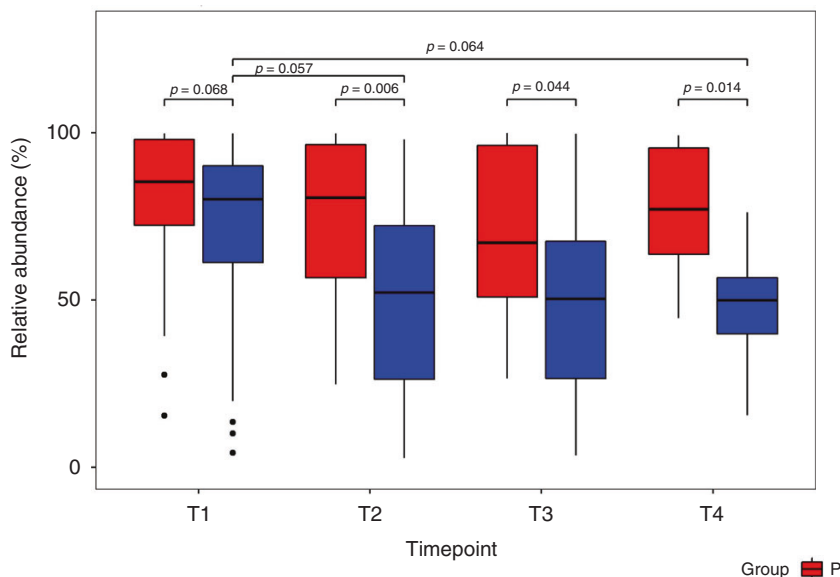

b Relative abundance of Bifidobacteriaceae family

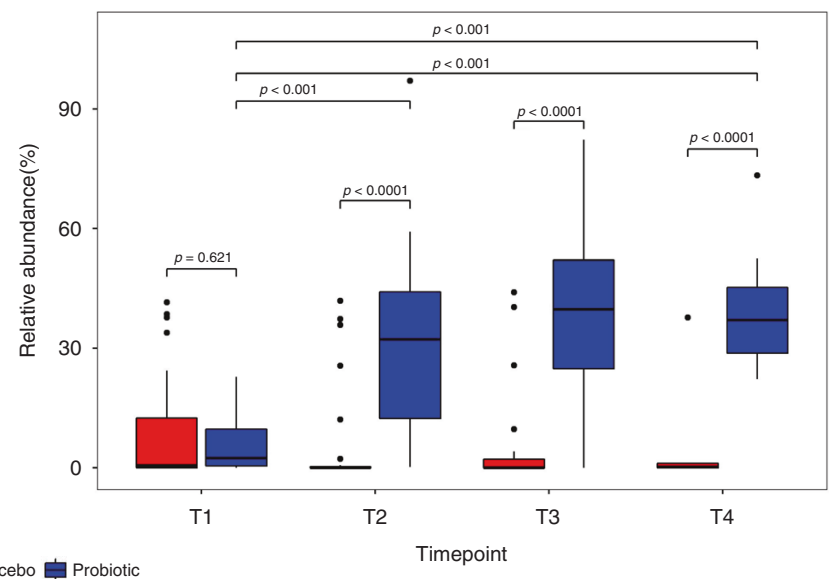

Fig. 3 Comparisons of relative abundances of bacterial families in the stool samples at various time points. a Comparison of relative abundance of potentially pathogenic families (sum-total of Clostridiaceae, Enterobacteriaceae, Enterococcaceae, Pseudomonadaceae, Staphylococcaceae, Streptococcaceae and Yersineaceae) between probiotic and placebo at various time points. At the bacterial family level, the relative abundance of the sum-total of potentially pathogenic families of Clostridiaceae, Enterobacteriaceae, Enterococcaceae, Pseudomonadaceae, Staphylococcaceae, Streptococcaceae \&Yersineaceae were significantly lower in the probiotic group compared to placebo at time points T2, T3, and T4 ( $p=0.002,0.033$, and 0.007 respectively). b Comparison of relative abundance of family Bifidobacteriaceae between probiotic and placebo at various time points. The relative abundance of the family Bifidobacteriaceae was significantly higher in the probiotic group at T2, T3, and T4 (all $p<0.001$ ).
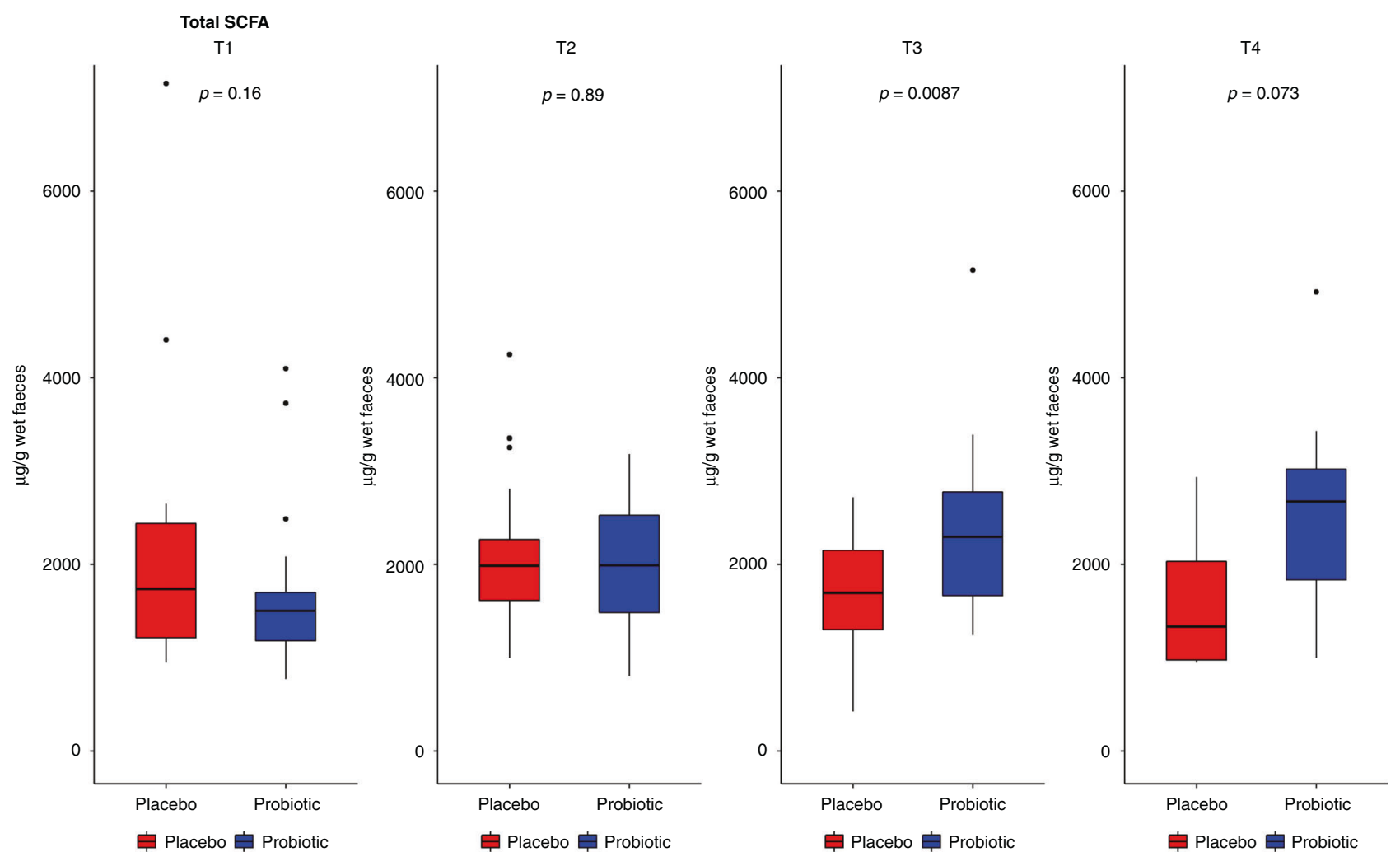

Fig. 4 Total SCFA levels in stools between probiotic and placebo groups at various time points. Total SCFA levels in stools were significantly higher in the probiotic group at time point T3 $(p=0.008)$. At other time points, the differences between the two groups were not statistically significant.

probiotic group, irrespective of the mode of delivery. However, the beneficial effects of supplementation on SCFA levels were significant only in infants born by caesarean section. The relative abundance of pathogenic families was not statistically significant in either mode of delivery, even though the trend was in favour of probiotics (Table 2).

\section{Clinical outcomes}

All participating infants survived. There were no significant differences between the groups in the incidence of HAl, duration of antibiotics, PN, hospital stay, and time to reach full feeds after surgery (Table 3). All routine clinical specimens (blood, urine, CSF, 
endotracheal secretions, wound swabs) from study infants were analysed using aerobic and anaerobic culture methods. There were no cases of infections due to the administered probiotics.

\section{Physical growth outcomes}

The z-scores for weight were significantly lower at discharge than at birth in the probiotic and placebo groups (both $p<0.0001$; e-Fig. 8 in Supplementary File 1). The degree of postnatal growth restriction for weight was similar between the two groups $(-0.93$ in the probiotic and -0.79 in the placebo; $p=0.486$; Table 1, e-Fig. 9 in Supplementary File 1). The $z$-scores for head circumference were lower at discharge than at birth in both the probiotic and placebo groups (both $p<0.0001$; e-Fig. 10 in Supplementary File 1). However, postnatal growth restriction for head circumference was less severe in the probiotic group ( $p=$ 0.013; Table 1 and Fig. 5). The $z$-scores for length at discharge were similar to those at birth in both the probiotic and placebo groups (e-Fig. 11). The degree of postnatal growth restriction for length was similar between the two groups (e-Fig. 12).

\section{DISCUSSION}

This pilot RCT found that after 2 weeks of supplementation with the three-strain bifidobacterial probiotic, neonates with CGISC had a lower relative abundance of potentially pathogenic bacterial families, higher abundance of bifidobacteria, and higher SCFA levels in their stools compared to placebo. Postnatal growth restriction for head circumference was less severe in the probiotic group than in the placebo group. Other clinical outcomes were similar between the two groups. No infections related to the administered probiotic organisms were observed. These results provide reassurance regarding the use of this probiotic in neonates with CGISC.

The only other RCT in neonates with surgical conditions was by Powell et al., ${ }^{22}$ in which 24 infants with gastroschisis were supplemented with Bifidobacterium longum subsp. infantis ATCC 15697 or placebo. In their study, the daily dose was $2 \times 10^{9} \mathrm{CFU}$ compared with $3 \times 10^{9} \mathrm{CFU}$ in our study. Similar to their study, the majority of our infants received breast milk as the sole source of diet, trial supplements were commenced in the postoperative period, and given for a median duration of 3 weeks. Similar to their study, the relative abundance of Bifidobacteriaceae increased to $40 \%$ after commencing supplements in the probiotic group and remained at $3 \%$ in the placebo group.

In line with the recent literature, our study found that neonates with CGISC develop postnatal growth restriction for weight and head circumference. ${ }^{29,30}$ Studies in preterm infants have reported an association between postnatal growth restriction of the head circumference and adverse developmental outcomes. ${ }^{31-33}$ Hence, it was reassuring that postnatal growth restriction for head circumference was less severe in the probiotic group in our RCT. Similar beneficial effects of probiotics on head circumference were observed in extremely low birth weight infants in recent RCTs. ${ }^{34,35}$

In our current RCT, SCFA levels were significantly higher in the probiotic group at T3 than in the placebo group, mainly due to elevated acetates. Even though the levels were also higher at T2 and T4, they were not statistically significant, probably because infants would have received only 1 week of supplementation by $\mathrm{T} 2$, whereas by T4, the sample size was very small. Bifidobacteria are known to ferment HMOs and produce acetate as a byproduct. ${ }^{36}$ Bifidobacteria are not butyrogenic, and hence butyrate levels were not elevated in the probiotic group in our study. While it has been suggested that acetate and lactate produced by bifidobacteria can be used as a substrate by other commensal bacteria (cross-feeding) ${ }^{37}$ to produce butyrate, thereby increasing its levels, this was not observed in our study.

The relative abundance of Proteobacteria, considered to be the microbial signature of gut dysbiosis, ${ }^{38}$ was lower in the probiotic 
Table 3. Clinical course and outcomes of study infants.

Mortality

\begin{tabular}{|c|c|c|}
\hline Probiotics & Placebo & $P$ values \\
\hline 0 & 0 & NE \\
\hline $4(13.3 \%)$ & $6(19.3 \%)$ & 0.731 \\
\hline 0 & $1(3 \%)$ & 1.000 \\
\hline 0 & $2(6.5 \%)$ & 0.492 \\
\hline 5 (IQR: 4-7) (range: 2-10) & 5 (IQR: 3-6) (range: 1-11) & 0.336 \\
\hline 3 (IQR: 0-6) (range: 0-31) & 2 (IQR: 0-5) (range: 0-13) & 0.305 \\
\hline $\begin{array}{l}9 \text { (IQR: 4-13) } \\
\text { (range: } 2-34 \text { ) }\end{array}$ & $\begin{array}{l}6 \text { (IQR: 5-10) } \\
\text { (range: 1-22) }\end{array}$ & 0.312 \\
\hline $1(3.3 \%)$ & $1(3.2 \%)$ & 1.000 \\
\hline 0 & 0 & NE \\
\hline $\begin{array}{l}13.9 \text { (IQR: 9-24) } \\
\text { (range: } 0-46)\end{array}$ & $\begin{array}{l}10.1 \text { (IQR: 6.9-21.3) } \\
\text { (range: } 0-58.7 \text { ) }\end{array}$ & 0.246 \\
\hline $30(100 \%)$ & $28(93.3 \%)$ & 0.492 \\
\hline 3 (IQR: 2-5) (range: 0-10) & 2 (IQR: 1-4) (range: 0-9) & 0.023 \\
\hline $\begin{array}{l}12 \text { (IQR: 8-16) } \\
\text { (range: } 0-46 \text { ) }\end{array}$ & $\begin{array}{l}9 \text { (IQR: 6-15) } \\
\text { (range: 1-52) }\end{array}$ & 0.306 \\
\hline $24(80 \%)$ & $22(71 \%)$ & 0.554 \\
\hline $6(20 \%)$ & $9(29 \%)$ & 0.554 \\
\hline $\begin{array}{l}27.5 \text { (IQR: 16-37) (range: } \\
10-102)\end{array}$ & $\begin{array}{l}20 \text { (IQR: 13-31) } \\
\text { (range: 10-67) }\end{array}$ & 0.094 \\
\hline-0.93 (SD 0.52) & -0.79 (SD 1.00) & 0.486 \\
\hline-0.20 (SD 0.63) & -0.70 (SD 0.84) & 0.011 \\
\hline-0.40 (SD 1.02) & -0.14 (SD 1.20) & 0.455 \\
\hline
\end{tabular}

Healthcare-associated infections (HABSI or UTI or SSI or VAP or pleural infection or peritonitis or meningitis or viral infection)

HABSI before commencing trial supplements

HABSI after commencing trial supplements

Duration of antibiotics before commencement of trial supplements (days)

Duration of antibiotics afterlcommencement of trial supplements (days)

Total duration of antibiotics (days)

Cholestatic jaundice

Sepsis due to the administered probiotic organism

Duration of PN (days)

EBM to commence feeds

Time to commence feeds after surgery (days)

Time to full enteral feeds after surgery (days)

Exclusive EBM at discharge

Use of formula milk

Duration of hospital stay (days)

Z-scores for weight at discharge minus at birth

$Z$-scores for head circumference at discharge minus at birth

$-0.40(S D 1.02)$

-0.14 (SD 1.20)

0.455

Bold values indicate statistical significance $p<0.05$

EBM expressed breast milk, NE not estimable, HABSI healthcare-associated bloodstream infections, UTI urinary tract infection, SSI surgical site infection, VAP ventilator-associated pneumonia, EBM expressed breast milk, IQR interquartile range, $N E$ not estimable, $S D$ standard deviation.

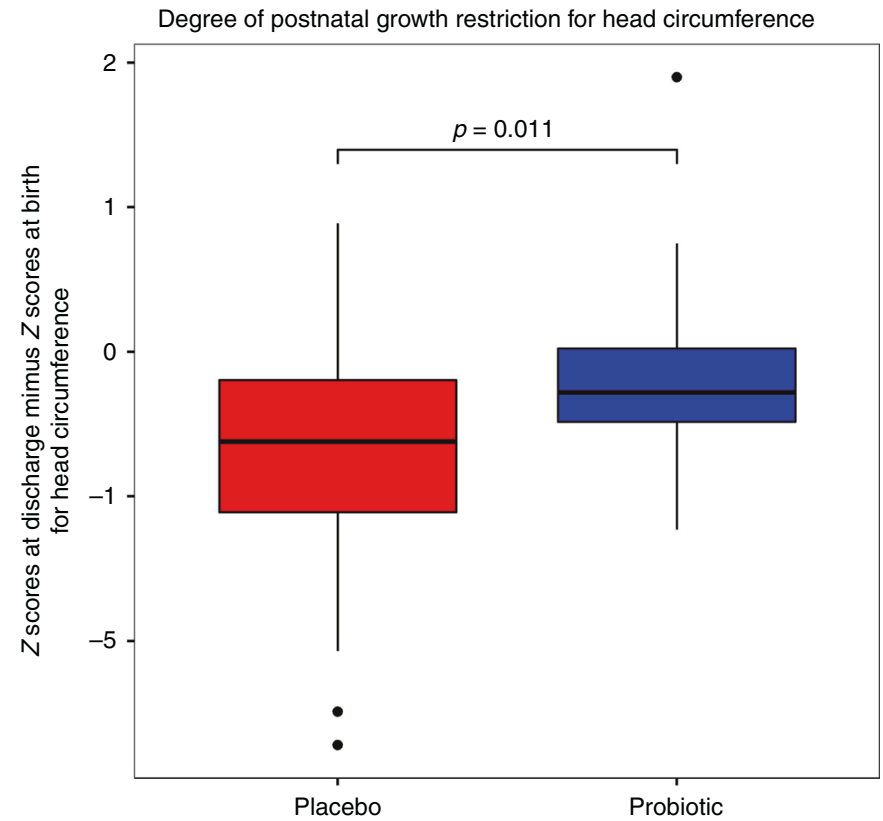

Fig. 5 Comparison of the degree of postnatal growth restriction for head circumference in study infants. The degree of postnatal growth restriction for head circumference was less severe in the probiotic group than in the placebo group. group than in the placebo group (22.7\% versus $50.3 \%)$, but the differences were not statistically significant. Studies with larger sample sizes are needed to determine if this difference is real.

Although not statistically significant, hospital stay was longer in the probiotic group ( 27 versus 20 days, $p=0.095$ ). We speculate that this was because the probiotic group had more infants with complex conditions such as $\mathrm{CDH}$ and gastroschisis, and fewer infants with imperforate anus.

It was reassuring that none of the infants developed infection due to the administered bifidobacterial strains. There are case reports of bifidobacterial sepsis in surgical infants and preterm infants without surgical conditions. ${ }^{39,40}$ Hence, ongoing microbiological surveillance is important when conducting probiotic RCTs.

In our study infants, the use of exclusive breast milk dropped to $70-80 \%$ by discharge compared to $93-100 \%$ at birth. However, the use of formula milk was similar between the two groups $(20 \%$ in the probiotic group versus $29 \%$ in the placebo group; $p=$ 0.554). The common reasons for the use of formula milk were inadequate production of breast milk and mother's choice. Some studies have suggested that probiotics are more effective in infants who are breastfed rather than formula. ${ }^{41-43}$ Given such findings and overall benefits of breast milk, every effort should be made to encourage breastfeeding and to express breast milk during hospital stay and after discharge in these infants.

While our study did not specifically address the issue of crosscontamination (aka cross-colonisation), ${ }^{44}$ the relative abundance of the genus Bifidobacterium in the placebo group was only $5 \%$ at all time points $\mathrm{T} 2-\mathrm{T} 4$, versus $35-45 \%$ in the probiotic group. Hence, even if there was cross-contamination, the load was not 
enough to allow them to colonise adequately in the placebo group and, hence, unlikely to be clinically significant.

An important limitation of our study was the higher rates of caesarean delivery in the placebo group. Infants born by caesarean delivery are known to have a lower abundance of bifidobacteria in the first few months of life. ${ }^{45}$ In this context, it was reassuring to know that even in subgroup analysis, the probiotic supplemented group had higher bifidobacterial counts. Our findings are similar to Frese et al. ${ }^{46}$ who reported that supplementation resulted in higher colonisation with bifidobacteria, which persisted even after supplementation was ceased, whether the infants were born vaginally or by caesarean section.

Since many factors such as gestational diabetes, ${ }^{47}$ mode of delivery, ${ }^{45}$ intrapartum antibiotics ${ }^{48-50}$ and maternal probiotics ${ }^{51-53}$ can affect neonatal gut microbiota, it is difficult to achieve a balance between the two groups for all such variables in small RCTs. Future studies should consider using the technique of "allocation by minimisation" ${ }^{\prime 14}$ to ensure adequate balance. In addition to maternal factors, important neonatal variables to be balanced include gestational age $\mathrm{e}^{55}$ and underlying surgical conditions. Since the type of milk used (breast milk versus cow's milk based versus hydrolysed formula) can influence gut microbiota, ${ }^{56}$ standardising their feeding regimen is desirable, but may not be feasible, given that multiple factors can influence milk production and its content. One option is to use pasteurised human donor milk, but the quantity required will be enormous because majority of these infants are born at term or near term, rather than at an extremely preterm gestation, in whom the quantity required is small. RCTs with large sample size have the potential to minimise the risk of imbalance in two groups regarding the type of milk used and other variables.

Another limitation of our study was that 16S rRNA gene sequencing allowed the allocation of reads only up to the genus level. Hence, it was not possible to confirm whether the increase in the relative abundance of Bifidobacterium in the probiotic group was due to the administered strains. A whole-metagenome approach that provides species- and strain-level information needs to be incorporated in future studies. Another limitation of the study was that stool samples from only 39 of the 61 study infants were available for analysis at T3, because others were discharged home by then.

While surgical conditions included in our RCT were heterogeneous, all of them had common issues such as feed intolerance, need for PN, and risk of infections. Hence, we decided to include all types of gastrointestinal surgical conditions in this pilot RCT. Another reason was because it would have taken at least 7-9 years to achieve the sample size of 60 if we had conducted it in a single surgical condition (for example, our unit admits only 10 cases of gastroschisis per year).

There is some observational evidence from adult literature that maltodextrin may lead to adverse health outcomes secondary to alterations in gut microbiota. ${ }^{57}$ In our RCT, the probiotic supplement contained probiotic organisms and maltodextrin, whereas placebo was only maltodextrin. Hence, any effect of maltodextrin on gut microbiota would have occurred in both placebo and probiotic groups. To evaluate the effects of maltodextrin (at the small dose of 1 gram per day as used in our trial) on gut microbiota, prospective cohort studies comparing gut microbiota in healthy breastfed infants receiving maltodextrin versus healthy breastfed infants are needed.

The important strengths of our study are as follows: (a) It is the first RCT to evaluate the efficacy and safety of the three-strain bifidobacterial supplementation in CGISC in neonates and (b) all study infants had baseline stool samples prior to commencing supplements.

The current pilot RCT found that the degree of postnatal growth restriction of head circumference was less severe in the probiotic supplemented group than placebo. Hence, one could hypothesise that probiotic supplemented group will have better neurodevelopmental outcomes. Our recent retrospective study ${ }^{30}$ with a sample size of 400 found the incidence of suboptimal neurodevelopmental outcomes (SNDO) to be $16 \%$ in term and nearterm neonates with CGISC. A sample size of 516 infants ( 258 in each arm) will be required to have $80 \%$ power at the two-sided $5 \%$ significance level to detect a $50 \%$ difference in the primary outcome ( $16 \%$ in controls and $8 \%$ in the probiotic group). Since nearly $30 \%$ of them are expected to be discharged home even before completing 2 weeks of supplementation, the sample size should be increased by another 154, and hence the final sample size will be around 670 infants. With such a large sample size, baseline frequencies of potential confounders such as gestational age, maternal antibiotics, maternal probiotic usage, mode of delivery, severity of illness and type of milk feeds are expected to be balanced between the two groups. Involvement of multiple centres will be crucial to achieve this sample size within a reasonable time period of 24-36 months.

In summary, this pilot RCT found that after 2 weeks of supplementation with the three-strain Bifidobacterium, neonates with CGISC had a lower relative abundance of potentially pathogenic bacterial families, higher abundance of Bifidobacterium, and higher SCFA levels in their stools. Larger studies with clinical endpoints and long-term follow-ups are necessary.

\section{REFERENCES}

1. Auber, F. et al. Enteric nervous system impairment in gastroschisis. Eur. J. Pediatr. Surg. 23, 29-38 (2013).

2. Bairdain, S. et al. A modern cohort of duodenal obstruction patients: predictors of delayed transition to full enteral nutrition. J. Nutr. Metab. 2014, 850820 (2014).

3. Smith, N. Oesophageal atresia and tracheo-oesophageal fistula. Early Hum. Dev. 90, 947-950 (2014).

4. Chung, E. Y. \& Yardley, J. Are there risks associated with empiric acid suppression treatment of infants and children suspected of having gastroesophageal reflux disease. Hosp. Pediatr. 3, 16-23 (2013).

5. Schwartz, M. Z. Novel therapies for the management of short bowel syndrome in children. Pediatr. Surg. Int. 29, 967-974 (2013).

6. Bower, T. R., Pringle, K. C. \& Soper, R. T. Sodium deficit causing decreased weight gain and metabolic acidosis in infants with ileostomy. J. Pediatr. Surg. 23, 567-572 (1988).

7. Mansour, F., Petersen, D., De Coppi, P. \& Eaton, S. Effect of sodium deficiency on growth of surgical infants: a retrospective observational study. Pediatr. Surg. Int. 30, 1279-1284 (2014).

8. Ng, D. H., Pither, C. A., Wootton, S. A. \& Stroud, M. A. The 'not so short-bowel syndrome': potential health problems in patients with an ileostomy. Colorectal Dis. 15, 1154-1161 (2013).

9. Demehri, F. R., Halaweish, I. F., Coran, A. G. \& Teitelbaum, D. H. Hirschsprungassociated enterocolitis: pathogenesis, treatment and prevention. Pediatr. Surg. Int. 29, 873-881 (2013).

10. Macfarlane, S. Antibiotic treatments and microbes in the gut. Environ. Microbiol. 16, 919-924 (2014).

11. Hallab, J. C. et al. Molecular characterization of bacterial colonization in the preterm and term infant's intestine. Indian J. Pediatr. 80, 1-5 (2013).

12. Fouhy, F. et al. High-throughput sequencing reveals the incomplete, short-term recovery of infant gut microbiota following parenteral antibiotic treatment with ampicillin and gentamicin. Antimicrob. Agents Chemother. 56, 5811-5820 (2012).

13. Rao, S. C. et al. Gut microbiota in neonates with congenital gastrointestinal surgical conditions: a prospective study. Pediatr. Res. 88, 878-886 (2020).

14. $\mathrm{Ma}$, J. et al. Comparison of gut microbiota in exclusively breast-fed and formulafed babies: a study of 91 term infants. Sci. Rep. 10, 15792 (2020).

15. Dahlgren, A. F. et al. Longitudinal changes in the gut microbiome of infants on total parenteral nutrition. Pediatr. Res. 86, 107-114 (2019).

16. Rao, S. C. \& Patole, S. K. Probiotic research in neonates with congenital gastrointestinal surgical conditions-now is the time. Microb. Biotechnol. 12, 254-258 (2019).

17. Tan, J. et al. The role of short-chain fatty acids in health and disease. Adv. Immunol. 121, 91-119 (2014).

18. Nogal, A., Valdes, A. M. \& Menni, C. The role of short-chain fatty acids in the interplay between gut microbiota and diet in cardio-metabolic health. Gut Microbes 13, 1-24 (2021).

19. Tsukuda, N. et al. Key bacterial taxa and metabolic pathways affecting gut shortchain fatty acid profiles in early life. ISME J. 15, 2574-2590 (2021).

20. Kok, C. R. et al. Stool microbiome, $\mathrm{pH}$ and short/branched chain fatty acids in infants receiving extensively hydrolyzed formula, amino acid formula, or human milk through two months of age. BMC Microbiol 20, 337 (2020). 
21. Chowdhury, A. H. et al. Perioperative probiotics or synbiotics in adults undergoing elective abdominal surgery: a systematic review and meta-analysis of randomized controlled trials. Ann. Surg. 271, 1036-1047 (2020).

22. Powell, W. T. et al. Probiotic administration in infants with gastroschisis: a pilot randomized placebo-controlled trial. J. Pediatr. Gastroenterol. Nutr. 62, 852-857 (2016).

23. Rao, S., Simmer, K. \& Patole, S. Probiotic supplementation in neonates with major gastrointestinal surgical conditions: a systematic review. J. Matern. Fetal Neonatal Med. 31, 1517-1523 (2018).

24. Fenton, T. R. \& Kim, J. H. A systematic review and meta-analysis to revise the fenton growth chart for preterm infants. BMC Pediatr. 13, 59 (2013).

25. de Onis, M., Garza, C., Onyango, A. W. \& Rolland-Cachera, M. F. [Who growth standards for infants and young children]. Arch. Pediatr. 16, 47-53 (2009).

26. Chou, J. H., Roumiantsev, S. \& Singh, R. Peditools electronic growth chart calculators: applications in clinical care, research, and quality improvement. J. Med. Internet Res. 22, e16204 (2020).

27. Mandal, S. et al. Analysis of composition of microbiomes: a novel method for studying microbial composition. Microb. Ecol. Health Dis. 26, 27663 (2015).

28. Moher, D. et al. Consort 2010 explanation and elaboration: updated guidelines for reporting parallel group randomised trials. J. Clin. Epidemiol. 63, e1-e37 (2010).

29. Strobel, K. M. et al. Growth failure prevalence in neonates with gastroschisis: a statewide cohort study. J. Pediatr. 233, 112-118 e113 (2021).

30. Batta, V. et al. Early neurodevelopmental outcomes of congenital gastrointestinal surgical conditions: a single-centre retrospective study. BMJ Paediatr. Open 4 e000736 (2020).

31. Meyers, J. M. et al. Neurodevelopmental outcomes in postnatal growth-restricted preterm infants with postnatal head-sparing. J. Perinatol. 36, 1116-1121 (2016).

32. Neubauer, V., Griesmaier, E., Pehbock-Walser, N., Pupp-Peglow, U. \& Kiechl-Kohlendorfer, $\mathrm{U}$. Poor postnatal head growth in very preterm infants is associated with impaired neurodevelopment outcome. Acta Paediatr. 102, 883-888 (2013).

33. Lee, E. S. et al. Factors associated with neurodevelopment in preterm infants with systematic inflammation. BMC Pediatr. 21, 114 (2021).

34. Wejryd, E., Marchini, G., Frimmel, V., Jonsson, B. \& Abrahamsson, T. Probiotics promoted head growth in extremely low birthweight infants in a double-blind placebo-controlled trial. Acta Paediatr. 108, 62-69 (2019).

35. Guney Varal, I., Koksal, N., Ozkan, H., Bagci, O. \& Dogan, P. Potential use of multistrain synbiotics for improving postnatal head circumference. Pak. J. Med. Sci. 34 1502-1506 (2018).

36. Alcon-Giner, C. et al. Microbiota supplementation with bifidobacterium and Lactobacillus modifies the preterm infant gut microbiota and metabolome: an observational study. Cell Rep. Med. 1, 100077 (2020).

37. De Vuyst, L. \& Leroy, F. Cross-feeding between bifidobacteria and butyrateproducing colon bacteria explains bifdobacterial competitiveness, butyrate production, and gas production. Int. J. food Microbiol. 149, 73-80 (2011).

38. Shin, N. R., Whon, T. W. \& Bae, J. W. Proteobacteria: microbial signature of dysbiosis in gut microbiota. Trends Biotechnol. 33, 496-503 (2015).

39. Ohishi, A. et al. Bifidobacterium septicemia associated with postoperative probiotic therapy in a neonate with omphalocele. J. Pediatr. 156, 679-681 (2010).

40. Jenke, A., Ruf, E. M., Hoppe, T., Heldmann, M. \& Wirth, S. Bifidobacterium septicaemia in an extremely low-birthweight infant under probiotic therapy. Arch. Dis. Child. Fetal Neonatal Ed. 97, F217-F218 (2012).

41. Sung, V. et al. Lactobacillus reuteri to treat infant colic: a meta-analysis. Pediatrics 141, e20171811 (2018)

42. Repa, A. et al. Probiotics (Lactobacillus acidophilus and Bifidobacterium infantis) prevent Nec in Vlbw infants fed breast milk but not formula [corrected]. Pediatr. Res. 77, 381-388 (2015).

43. Dos Reis Buzzo Zermiani, A. P. et al. Evidence of Lactobacillus reuteri to reduce colic in breastfed babies: systematic review and meta-analysis. Complement Ther Med. 63, 102781 (2021)

44. Gengaimuthu, K. The cross contamination (cross colonization) phenomenon of probiotic use in neonatal intensive care units: putative mechanisms and clinical and research implications. Cureus 10, e2691 (2018).

45. Shaterian, N., Abdi, F., Ghavidel, N. \& Alidost, F. Role of cesarean section in the development of neonatal gut microbiota: a systematic review. Open Med. (Wars.) 16, 624-639 (2021)

46. Frese, S. A. et al. Persistence of supplemented Bifidobacterium longum subsp. infantis Evc001 in breastfed infants. mSphere 2, e00501-17 (2017).

47. Crusell, M. K. W. et al. Comparative studies of the gut microbiota in the offspring of mothers with and without gestational diabetes. Front. Cell Infect. Microbiol. 10, 536282 (2020).

48. Zimmermann, P. \& Curtis, N. Effect of intrapartum antibiotics on the intestinal microbiota of infants: a systematic review. Arch. Dis. Child. Fetal Neonatal Ed. 105, 201-208 (2020).
49. Nogacka, A. et al. Impact of intrapartum antimicrobial prophylaxis upon the intestinal microbiota and the prevalence of antibiotic resistance genes in vaginally delivered full-term neonates. Microbiome 5, 93 (2017).

50. Wong, W. S. W. et al. Prenatal and peripartum exposure to antibiotics and cesarean section delivery are associated with differences in diversity and composition of the infant meconium microbiome. Microorganisms 8 (2020).

51. Dotterud, C. K. et al. Does maternal perinatal probiotic supplementation alter the intestinal microbiota of mother and child? J. Pediatr. Gastroenterol. Nutr. 61 200-207 (2015)

52. Gueimonde, M. et al. Effect of maternal consumption of Lactobacillus Gg on transfer and establishment of fecal bifidobacterial microbiota in neonates. J. Pediatr. Gastroenterol. Nutr. 42, 166-170 (2006).

53. Zaidi, A. Z., Moore, S. E. \& Okala, S. G. Impact of maternal nutritional supplementation during pregnancy and lactation on the infant gut or breastmilk microbiota: a systematic review. Nutrients 13, 1137 (2021).

54. Altman, D. G. \& Bland, J. M. Treatment allocation by minimisation. BMJ 330, 843 (2005).

55. Chernikova, D. A. et al. The premature infant gut microbiome during the first 6 weeks of life differs based on gestational maturity at birth. Pediatr. Res $\mathbf{8 4}$ 71-79 (2018)

56. Azad, M. B. et al. Impact of maternal intrapartum antibiotics, method of birth and breastfeeding on gut microbiota during the first year of life: a prospective cohort study. BJOG 123, 983-993 (2016).

57. Nickerson, K. P. Chanin, R. \& McDonald, C. Deregulation of intestinal antimicrobial defense by the dietary additive, maltodextrin. Gut Microbes 6, 78-83 (2015)

\section{ACKNOWLEDGEMENTS}

We sincerely thank the following: Dr. Fumiaki Abe and Dr. Noriyuki Iwabuchi of Morinaga Milk Industry Co., Ltd, Japan, for providing trial supplements free of cost Ms. Margaret Shave and Ms. Thanh Tan for help with receiving the trial supplements, generating random sequence numbers, providing trial supplements for administration to study infants, and for maintaining audits. Members of the Data Safety and Monitoring Committee: Prof. Tobias Strunk (neonatologist) and Mr. Naeem Samnakay (paediatric surgeon). Medical scientific staff at PathWest Laboratory Medicine WA for performing microbial analysis of sachets. The nursing staff at Perth Children's Hospital for collecting stool samples and administering trial supplements to study infants. Mr. Damber Shrestha for providing data from the neonatal database. Ms. Emma Anderton, Ms. Julie Hibbert, and Ms. Jamee Murdoch for processing the stool samples and storing them in a deep freezer at $-80^{\circ} \mathrm{C}$. Ms. Yen Kok for helping with organising the shipment of stool samples to the lab at the University of New South Wales. Centre for Neonatal Research and Education, Neonatal Directorate, King Edward Memorial Hospital for Women, Western Australia for providing funds. Parents of study infants for providing informed consent for participation in the RCT. Study infants for participating in the RCT. Centre for Neonatal Research and Education, Neonatal Directorate, Child and Adolescent Health Service, Western Australia.

\section{AUTHOR CONTRIBUTIONS}

Substantial contributions to conception and design, acquisition of data, or analysis and interpretation of data, drafting the article or revising it critically for important intellectual content, and final approval of the version to be published: S.C.R., M.E., L.C., A.D.K., I.J.G., K.N.S., B.W., P.L.C., and S.K.P.

\section{COMPETING INTERESTS}

The authors declare no competing interests.

\section{ADDITIONAL INFORMATION}

Supplementary information The online version contains supplementary material available at https://doi.org/10.1038/s41390-021-01884-x.

Correspondence and requests for materials should be addressed to Shripada Rao.

Reprints and permission information is available at http://www.nature.com/ reprints

Publisher's note Springer Nature remains neutral with regard to jurisdictional claims in published maps and institutional affiliations. 